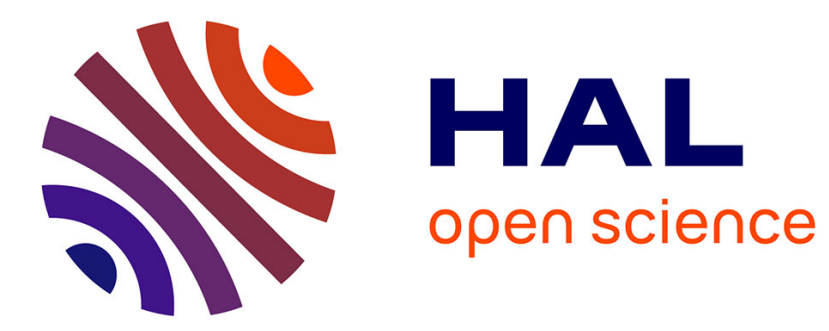

\title{
Influence d'un gaz tampon sur la durée de vie d'ions confinés
}

\author{
M. Vedel
}

\section{To cite this version:}

M. Vedel. Influence d'un gaz tampon sur la durée de vie d'ions confinés. Journal de Physique Lettres, 1976, 37 (12), pp.339-340. 10.1051/jphyslet:019760037012033900 . jpa-00231306

\section{HAL Id: jpa-00231306 https://hal.science/jpa-00231306}

Submitted on 1 Jan 1976

HAL is a multi-disciplinary open access archive for the deposit and dissemination of scientific research documents, whether they are published or not. The documents may come from teaching and research institutions in France or abroad, or from public or private research centers.
L'archive ouverte pluridisciplinaire HAL, est destinée au dépôt et à la diffusion de documents scientifiques de niveau recherche, publiés ou non, émanant des établissements d'enseignement et de recherche français ou étrangers, des laboratoires publics ou privés. 


\title{
INFLUENCE D'UN GAZ TAMPON SUR LA DURÉE DE VIE D'IONS CONFINÉS
}

\author{
M. VEDEL \\ Interactions Ioniques, Université de Provence, \\ Centre de St-Jérôme, 13397 Marseille Cedex 4, France
}

(Reçu le 15 juillet 1976, accepté le 5 octobre 1976)

\begin{abstract}
Résumé. - On mesure la durée de vie d'ions Xénon confinés par un piège quadrupolaire en fonction de la densité d'un gaz tampon. Les résultats sont en accord avec un modèle théorique de collision à sphères dures.
\end{abstract}

Abstract. - Lifetimes of ions confined by a radio frequency quadrupole field in the presence of a buffer gas are measured. The results are in good agreement with a hard sphere collision model.

Des observations antérieures ont montré l'influence d'un gaz léger, volontairement introduit, sur la durée de vie d'ions confinés par un champ quadrupolaire radiofréquence [1].

Dans une première approche du phénomène on considère les collisions des ions d'une part avec les atomes dont ils sont issus et qui ont globalement pour effet de diminuer le temps de confinement et d'autre part avec les atomes du gaz tampon. Une étude théorique a été effectuée sur un modèle monodimensionnel [2, 3, 4]. Il s'en dégage en particulier l'existence d'un domaine de pression du gaz tampon favorable à l'augmentation des propriétés de confinement du piège. Les résultats expérimentaux que nous rapportons ici sont les premiers d'une étude systématique et permettent de contrôler la validité du modèle.

On utilise un quadrupôle cylindrique plus facile à réaliser qu'une structure hyperboloïque, dont les paramètres géométriques sont

$$
R_{0}=\sqrt{2} Z_{0}=1,41 \mathrm{~cm} .
$$

La fréquence de confinement est de $278,00 \mathrm{kHz}$ et la tension appliquée de l'ordre de $60 \mathrm{~V}$ efficaces. Ceci correspond pour les ions Xénon à une énergie totale maximale de $6,4 \mathrm{eV}$. Les ions sont créés par bombardement électronique à partir d'une pression partielle de Xénon de quelques $10^{-9}$ torr. Le pompage est assuré par une pompe ionique de $501 / \mathrm{s}$ équipée d'éléments de pompage de gaz rares. On utilise comme gaz tampon de l'hélium que l'on fait diffuser dans le montage au moyen d'une fuite à quartz. La mesure du nombre d'ions se fait suivant la méthode paramétrique décrite par Dehmelt [5].
Cette méthode présente l'intérêt de séparer les fréquences d'excitation et de détection; le signal se trouve alors peu sensible aux fluctuations de la tension d'excitation. La fréquence d'excitation est de $317,71 \mathrm{kHz}$ ce qui correspond au passage à la résonance, à une fréquence du mouvement séculaire de $39,71 \mathrm{kHz}$; c'est sur celle-ci qu'est accordé le circuit de mesure. Le signal produit par le mouvement cohérent est amplifié, redressé et enregistré après passage dans un détecteur crête.

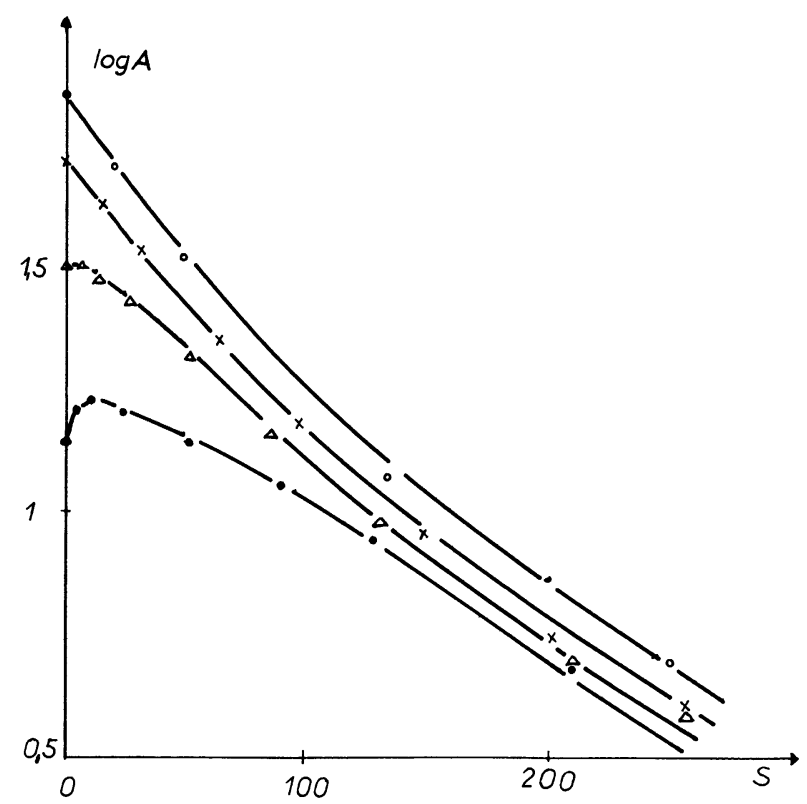

FIG. 1. - Variation de l'amplitude du signal de résonance pour différentes valeurs du nombre initial d'ions. Conditions expérimentales : expression de Xénon de l'ordre de $3 \times 10^{-9}$ torr, pression d'hélium $3 \times 10^{-9}$ torr; $s$ est le temps en seconde. 
L'amplitude du signal de résonance est liée à la répartition énergétique des ions dans la cage [6]. Il faut atteindre la thermalisation de la population, ce qui correspond à un groupement autour du centre de symétrie du système, pour qu'il y ait proportionnalité entre le signal observé et le nombre d'ions. Cette thermalisation est réalisée au bout d'un temps qui constitue pour le système d'ions, le temps de perte de mémoire des conditions initiales de création. La figure 1 illustre l'évolution de la grandeur du signal mesuré au cours du temps pour différentes valeurs du nombre initial d'ions et dans des conditions de pressions identiques. Pour ces conditions de collisions la direction asymptotique, indépendante des conditions initiales est atteinte au bout de 100 secondes.

La figure 2 représente la variation des durées de vie asymptotiques de l'ion Xénon en fonction de la pression d'hélium dans un mélange ternaire $\mathrm{Xe}^{+}-\mathrm{Xe}-\mathrm{He}$. Ces conditions expérimentales sont

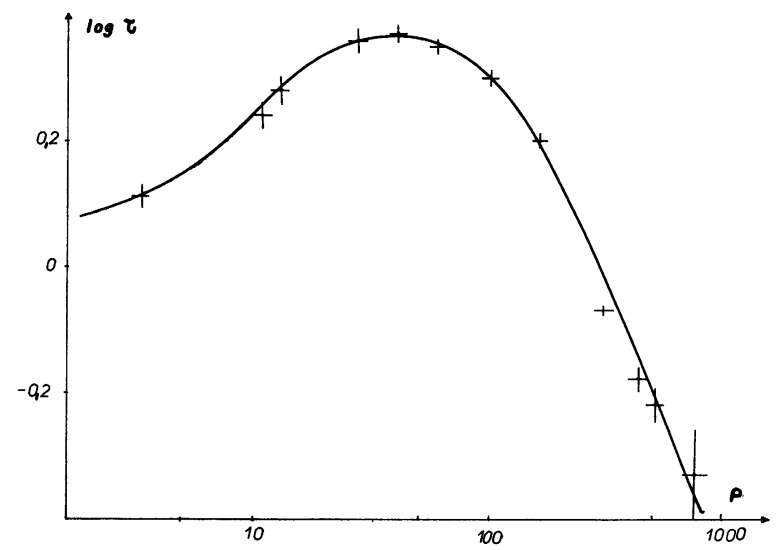

FIG. 2. - Variation de la durée de vie de l'ion Xénon en fonction de la pression d'hélium pour un mélange ternaire $\mathrm{Xe}^{+}, \mathrm{Xe}, \mathrm{He}$ avec une pression partielle de Xe de $3 \times 10^{-9}$ torr; $\omega_{0} Z_{0}=2500 \mathrm{~m} / \mathrm{s}$ où $\omega_{0}$ est la pulsation du mouvement séculaire et $Z_{0}$ la hauteur de la cage. voisines de celles prises en compte dans le modèle théorique (Fig. 3) où l'on considère le mélange ternaire $\mathrm{C}_{\mathrm{S}}^{+}-\mathrm{Xe}-\mathrm{He}$ : masses des ions semblables et même ordre de grandeur de l'énergie maximale. Les résultats expérimentaux confirment l'existence d'un domaine de pression du gaz tampon qui rend maximales les propriétés de confinement. La variation de la durée de vie mesurée est cependant moins importante que celle prévue théoriquement [4]. Ceci est dû essentiellement au fait que le modèle utilisé est un modèle de collision à sphères dures, monodimensionnel où n'interviennent ni taux de fuite naturel, ni charge d'espace.

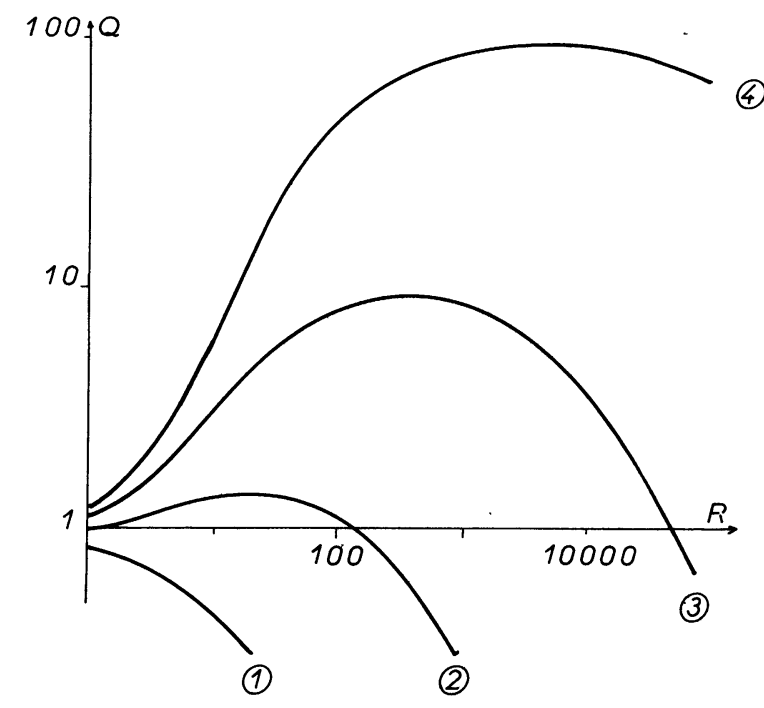

FIG. 3. - Variation du facteur d'amélioration $Q$ de la durée du confinement pour différentes valeurs de l'énergie maximale $\omega_{0} Z_{0}$ des ions. En 1 (resp. 2, 3, 4), $\omega_{0} Z_{0}=200$ (resp. 400, 600, 800) $\mathrm{ms}^{-1}$, et pour un mélange ternaire $\mathrm{C}_{\mathrm{S}}^{+}-\mathrm{Xe}-\mathrm{He}$ [4]. $Q$ est défini par $\tau_{1} / \tau_{2}$ où $\tau_{1}$ est la durée de vie de $\mathrm{C}_{S}^{+}$dans un mélange ternaire $\mathrm{C}_{\mathrm{S}}^{+}-\mathrm{Xe}-\mathrm{He}$, et $\tau_{2}$ la durée de vie de $\mathrm{C}_{\mathrm{S}}^{+}$dans le mélange binaire $\mathrm{C}_{\mathrm{S}}^{+}-\mathrm{C}_{\mathrm{S}}, R$ représente $P_{3} / \sigma_{3} / P_{1} / \sigma_{1}$ où $P_{3}$ (resp. $P_{1}$ ) est la pression partielle de $\mathrm{He}$ (resp. Xe) et $\sigma_{3}$ (resp. $\sigma_{1}$ ) la section efficace totale de collision élastique de $\mathrm{C}_{\mathrm{S}}^{+}$dans $\mathrm{He}$ (resp. $\mathrm{C}_{\mathrm{S}}^{+}$dans $\mathrm{C}_{\mathrm{S}}$ ).

\section{Bibliographie}

[1] Dawson, P. H., Whetten, N. R., Naturwissenschaften 56 (1969) 109.

[2] André, J., Schermann, J. P., Phys. Lett. 45A (1973) 139.

[3] ANDré, J., Vedel, F., Physica 81C (1976) 376.
[4] ANDRÉ, J., J. Physique 37 (1976) 719.

[5] Schuessler, H. A., Fortson, E. N., Dehmelt, H. G., Phys. Rev. 187 (1969) 5-38.

[6] MAJOR, G., Doctoral Thesis. University of Washington (1962). 